\title{
Tests for quantum contextuality in terms of $q$-entropies
}

\author{
Alexey E. Rastegin* \\ Department of Theoretical Physics, Irkutsk State University, Gagarin Bv. 20, Irkutsk 664003, Russia
}

\begin{abstract}
The information-theoretic approach to Bell's theorem is developed with use of the conditional $q$ entropies. The $q$-entropic measures fulfill many similar properties to the standard Shannon entropy. In general, both the locality and noncontextuality notions are usually treated with use of the so-called marginal scenarios. These hypotheses lead to the existence of a joint probability distribution, which marginalizes to all particular ones. Assuming the existence of such a joint probability distribution, we derive the family of inequalities of Bell's type in terms of conditional $q$-entropies for all $q \geq 1$. Quantum violations of the new inequalities are exemplified within the Clauser-Horne-ShimonyHolt (CHSH) and Klyachko-Can-Binicioğlu-Shumovsky (KCBS) scenarios. An extension to the case of $n$-cycle scenario is briefly mentioned. The new inequalities with conditional $q$-entropies allow to expand a class of probability distributions, for which the nonlocality or contextuality can be detected within entropic formulation. The $q$-entropic inequalities can also be useful in analyzing cases with detection inefficiencies. Using two models of such a kind, we consider some potential advantages of the $q$-entropic formulation.
\end{abstract}

PACS numbers:

Keywords: Bell theorem, contextuality hypothesis, marginal scenario, conditional $q$-entropy, chain rule

\section{INTRODUCTION}

The notion of entanglement plays a key role in studies of non-classical features of quantum theory. Due to impressive advances, entangled quantum states are now treated as tools for information processing [1]. An existence of purely quantum correlations was emphasized in the Schrödinger "cat paradox" paper [2] and the Einstein-Podolsky-Rosen paper [3]. Nonlocal correlations are brightly manifested in specified experiments similar to Bohm's version of the EPR argument [4]. In such experiments, spacelike separated observers share subsystems of an entangled quantum system. From an intuitive viewpoint, the following assumptions seem to be relevant. First, one assumes that physical quantities have well established values previous to any measurement. Second, no signals can travel faster than the speed of light. A less known point is the assumption of measurement independence [ $\underline{5},[\underline{6}$ ]. The assumptions lead to restrictions commonly referred to as Bell inequalities. The fundamental result is that such restrictions on correlations are overcome within quantum mechanics [7]. Today, a role of Bell inequalities widely ranges from the foundations [8] up to applications in quantum information processing like quantum key distillation [9, 10] and randomness expansion [11]. In a certain sense, Leggett-Garg inequalities [12] are closely related to Bell ones. On the other hand, Leggett-Garg inequalities probe correlations of a single system measured at different times. A theoretical background, experimental tests and some proposals for such inequalities are reviewed in Ref. [13]. In Ref. [14], this issue is examined within the entropic approach.

Like the locality, the noncontextuality assumption is also natural from the classical viewpoint. In quantum theory, this pertains only to mutually compatible observables, which are simultaneously diagonalizable. Hence, performed measurement of one of such observables does not stipulate results of further measurements of other. It turns out that no noncontextual hidden-variable models can reproduce all the predictions of quantum theory [15, 16]. This result known as the Kochen-Specker theorem was independently obtained by Bell (for details, see Ref. 17]). The recent paper [18] focused on the causality, which is also deeply rooted in our understanding of the macro world. In quantum mechanics, we may conceive situations in which a single event can be equally a cause and an effect of another one [18]. As discussed results concern measurement statistics, they are statements about probability distributions. In general, there are various ways to express probabilistic properties. Although many formulations of Bell's theorem use inequalities, the Greenberger-Horne-Zeilinger argument has provided a claim without inequalities [19]. The EPR and GHZ states can give suitable tools in considering three-partite entanglement 20]. Bell inequalities can be treated geometrically within multilinear-contraction framework [21]. Entropic formulations of Bell's theorem have been proposed in Ref. [22] and further examined in Ref. [23]. Various entropic measures are indispensable tools in analyzing secure protocols [24].

*Electronic address: rast@api.isu.ru; alexrastegin@mail.ru 
There exist several concrete scenarios to realize Bell's theorem as an experimentally tested statement. The ClauserHorne-Shimony-Holt (CHSH) scenario [25] is probably the most known setup of such a kind. The CHSH inequality imposes a restriction on mean values of the corresponding observables. Its violation allows to renounce local hiddenvariable models [26, 27]. The Klyachko-Can-Binicioğlu-Shumovsky (KCBS) scenario [28] is also the subject of active research. The entropic approach has been applied to both the CHSH [22, 29] and KCBS scenarios [29, 30]. These scenarios can be treated respectively as the $n=4$ and $n=5$ cases of more general $n$-cycle scenario [31, 32]. For the $n$-cycle scenario, the quantum violations occur for all $n$, though technical motives make their observation harder for large $n$ [33]. Various aspects of entropic inequalities for marginal problems are considered in Ref. [34]. The information-theoretical results are usually expressed in terms of standard functionals based on the Shannon entropy. Applying statistical methods in numerous topics, some extensions were found to be useful. The Rényi [35] and Tsallis [36] entropies are both especially important generalizations. The nonlocality and contextuality are genuine quantum features related also to the field of quantum information processing. So, it is of importance to develop the entropic approach to Bell inequalities with use of generalized entropies.

The aim of the present paper is to study information-theoretic formulations of Bell's theorem in terms of the conditional Tsallis entropies. It turns out that important achievements can be reached in this way. The paper is organized as follows. In Sect. 2, basic properties of the Tsallis entropies are recalled. We also prove two required statements about the conditional $q$-entropy, one of them for $q \geq 1$ only. In Sect. 3, marginal scenarios are discussed from the viewpoint of their use in studying Bell inequalities. For the CHSH scenario, inequalities of Bell's type in terms of the conditional $q$-entropies are obtained in Sect. 4 . We also mention an extension to the $n$-cycle scenario, which is currently the subject of active research [31, 32]. In Sect. 5, we consider $q$-entropic inequalities with $q \geq 1$ for the KCBS scenario. In both the cases, violations of the obtained inequalities could be tested in the experiment. As is shown, $q$-entropic inequalities with suitably chosen $q>1$ can detect the nonlocality or contextuality of some probability distributions, for which inequalities with the standard entropy fail. We also analyze the $q$-entropic inequalities within two models of detection inefficiencies. In other words, the family of $q$-entropic inequalities is much more powerful to reveal such properties. In Sect. 6, we conclude the paper with a summary of results.

\section{CONDITIONAL $q$-ENTROPIES AND THEIR PROPERTIES}

In this section, we recall definitions of the Tsallis entropies and related conditional entropies. Required properties of these entropic functionals are discussed as well. Let the variable $A$ take values on the set $\Omega_{A}$ with corresponding probability distribution $\left\{p(a): a \in \Omega_{A}\right\}$. The Tsallis entropy of order $q>0 \neq 1$ is defined by [36]

$$
H_{q}(A):=\frac{1}{1-q}\left(\sum_{a \in \Omega_{A}} p(a)^{q}-1\right) .
$$

With the factor $\left(2^{1-q}-1\right)^{-1}$ instead of $(1-q)^{-1}$, this entropic form was derived from several axioms by Havrda and Charvát [37]. Let $B$ be another variable taking values on the set $\Omega_{B}$ with probability distribution $\left\{p(b): b \in \Omega_{B}\right\}$. The joint $q$-entropy $H_{q}(A, B)$ is defined similarly to Eq. (1), but with joint probabilities $p(a, b)$ instead of $p(a)$. It is sometimes convenient to rewrite the entropy (11) as

$$
H_{q}(A)=-\sum_{a \in \Omega_{A}} p(a)^{q} \ln _{q} p(a)=\sum_{a \in \Omega_{A}} p(a) \ln _{q} \frac{1}{p(a)} .
$$

The $q$-logarithm $\ln _{q} x=\left(x^{1-q}-1\right) /(1-q)$ is defined for $q>0 \neq 1$ and $x>0$, and it obeys $\ln _{q}(1 / x)=-x^{q-1} \ln _{q} x$. In the limit $q \rightarrow 1$, we obtain $\ln _{q} x \rightarrow \ln x$ and the standard Shannon entropy

$$
H_{1}(A)=-\sum_{a \in \Omega_{A}} p(a) \ln p(a)
$$

For brevity, we will usually omit the symbol of the set $\Omega_{A}$ in entropic sums. Properties of quantum counterpart of the entropy (11) are examined in Ref. [38]. Applications of various entropic functions in studying quantum systems are discussed in the book [39].

To analyze more realistic cases with detector inefficiencies, the following questions will rise. For the given $\eta \in[0 ; 1]$ and probability distribution $\left\{p(a): a \in \Omega_{A}\right\}$, the set

$$
\left\{p_{\eta}\right\}:=\left\{\eta p(a): a \in \Omega_{A}\right\} \cup\{1-\eta\}
$$


is a probability distribution as well. This probability distribution corresponds to some random variable $A_{\eta}$. We aim to relate the entropy $H_{q}\left(A_{\eta}\right)$ with $H_{q}(A)$ and the binary $q$-entropy

$$
h_{q}(\eta):=-\eta^{q} \ln _{q} \eta-(1-\eta)^{q} \ln _{q}(1-\eta)
$$

From three probability distributions, we can built another probability distribution

$$
\left\{p_{\eta \eta}\right\}:=\left\{\eta^{2} p(a)\right\} \cup\{\eta(1-\eta) p(b)\} \cup\{\eta(1-\eta) p(c)\} \cup\left\{(1-\eta)^{2}\right\} .
$$

In this case, we aim to relate the obtained $q$-entropy with the $q$-entropies of the initial probability distributions. The following statement takes place.

Lemma 1 Let random variable $A_{\eta}$ take its values according to the probability distribution (4). For all $q>0$, the q-entropies satisfy

$$
H_{q}\left(A_{\eta}\right)=\eta^{q} H_{q}(A)+h_{q}(\eta)
$$

Let random variable $A_{\eta \eta}$ take its values according to the probability distribution (6). For all $q>0$, the $q$-entropies satisfy

$$
H_{q}\left(A_{\eta \eta}\right)=\eta^{2 q} H_{q}(A)+\eta^{q}(1-\eta)^{q}\left(H_{q}(B)+H_{q}(C)\right)+\left(\eta^{q}+(1-\eta)^{q}+1\right) h_{q}(\eta)
$$

Proof. We first assume $q>0 \neq 1$. Substituting the distribution (41) into Eq. (11) directly leads to the formula

$$
(1-q) H_{q}\left(A_{\eta}\right)=\eta^{q}\left(\sum_{a} p(a)^{q}-1\right)+\eta^{q}+(1-\eta)^{q}-1
$$

Dividing Eq. (9) by $(1-q)$ gives the claim (7). Similarly to Eq. (9), we further write the term $(1-q) H_{q}\left(A_{\eta \eta}\right)$ as

$$
\eta^{2 q}\left(\sum_{a} p(a)^{q}-1\right)+\eta^{q}(1-\eta)^{q}\left(\sum_{b} p(b)^{q}+\sum_{c} p(c)^{q}-2\right)+\eta^{2 q}+2 \eta^{q}(1-\eta)^{q}+(1-\eta)^{2 q}-1
$$

By the identity $\eta^{2 q}+2 \eta^{q}(1-\eta)^{q}+(1-\eta)^{2 q}-1=\left(\eta^{q}+(1-\eta)^{q}+1\right)\left(\eta^{q}+(1-\eta)^{q}-1\right)$, we get the claim (8) from Eq. (10) after dividing by $(1-q)$. The standard case is recovered in the limit $q \rightarrow 1$.

The second summand in the right-hand side of Eq. (7) can easily be checked with any deterministic probability distribution. If the initial distribution $\{p(a)\}$ is deterministic, then the deformed distribution (4) includes only two nonzero probabilities, namely $\eta$ and $(1-\eta)$. As $H_{q}(A)=0$, the right-hand side of Eq. (7) actually gives the binary $q$-entropy. Similarly, the third summand in the right-hand side of Eq. (8) could be checked with three deterministic probability distributions.

Originally, the Braunstein-Caves inequality was formulated with use of the conditional entropy and its generic properties [22]. The writers of Ref. [23] derived entropic Bell inequalities by considering the so-called entropy Venn diagrams. The entropy of $A$ conditional on knowing $B$ is defined as [40]

$$
H_{1}(A \mid B):=\sum_{b} p(b) H_{1}(A \mid b)=-\sum_{a} \sum_{b} p(a, b) \ln p(a \mid b)
$$

where $H_{1}(A \mid b):=-\sum_{a} p(a \mid b) \ln p(a \mid b)$ and $p(a \mid b)=p(a, b) p(b)^{-1}$ according to the Bayes rule. The quantity (11) will be referred to as the standard conditional entropy. Further, we will use its $q$-entropic extension. By means of the particular functional

$$
H_{q}(A \mid b):=-\sum_{a} p(a \mid b)^{q} \ln _{q} p(a \mid b)=\sum_{a} p(a \mid b) \ln _{q} \frac{1}{p(a \mid b)}
$$

one defines the conditional $q$-entropy 41,42 ]

$$
H_{q}(A \mid B):=\sum_{b} p(b)^{q} H_{q}(A \mid b)
$$

In the limit $q \rightarrow 1$, this definition is reduced to Eq. (11). The above entropic measures with $q=2$ have been used in Ref. [44] for estimating the error probability on checking statistical hypotheses. Below, we will extensively use the following properties of the entropic function (13). For all $q>0$, the entropy (13) satisfies

$$
H_{q}(A, B)=H_{q}(B \mid A)+H_{q}(A)=H_{q}(A \mid B)+H_{q}(B)
$$


This formula expresses the chain rule for the conditional $q$-entropy [41]. It can easily be derived in line with the definitions (2) and (13) by means of the identity

$$
\ln _{q}(x y)=\ln _{q} x+x^{1-q} \ln _{q} y
$$

The mutual information is widely used in information theory [40]. Similarly to the standard case, the mutual $q$ information can be defined as [41]

$$
I_{q}(A: B):=H_{q}(A)-H_{q}(A \mid B) .
$$

For $q=1$, we have the standard mutual information $I_{1}(A: B)=H_{1}(A)-H_{1}(A \mid B)$. Using normalized Tsallis entropies, the corresponding mutual information was introduced in Ref. [45]. We can rewrite (16) in the form

$$
I_{q}(A: B)=I_{q}(B: A)=H_{q}(A)+H_{q}(B)-H_{q}(A, B),
$$

since $H_{q}(A \mid B)=H_{q}(A, B)-H_{q}(B)$ by Eq. (14). So, the quantity (16) is symmetric in its entries. Quantum violations of the Clauser-Horne-Shimony-Holt inequality is limited from above by the Tsirel'son bound [46]. This bound can be derived from the assumption that the chain rule holds for a generalized mutual information proposed in Ref. [47].

The chain rule (14) can further be extended to more than two variables. According to theorem 2.4 of Ref. [41], one obeys

$$
H_{q}\left(A_{1}, A_{2}, \ldots, A_{n}\right)=\sum_{j=1}^{n} H_{q}\left(A_{j} \mid A_{j-1}, \ldots, A_{1}\right)
$$

Using Eq. (14) and non-negativity of the conditional $q$-entropy, we immediately obtain

$$
H_{q}(A) \leq H_{q}(A, B), \quad H_{q}(B) \leq H_{q}(A, B) .
$$

In the next section, we will also use inequalities of the following form.

Lemma 2 For real $q \geq 1$ and integer $n \geq 1$, the conditional $q$-entropy satisfies

$$
H_{q}\left(A \mid B_{1}, \ldots, B_{n-1}, B_{n}\right) \leq H_{q}\left(A \mid B_{1}, \ldots, B_{n-1}\right) .
$$

Proof. Let us assume $q>1$. First, we prove the claim for $n=2$. The conditional $q$-entropy $H_{q}(A \mid B, C)$ can be rewritten as

$$
H_{q}(A \mid B, C)=\sum_{a b} p(b)^{q} \sum_{c}\left(\frac{p(b, c)}{p(b)}\right)^{q} f_{q}(p(a \mid b, c)),
$$

where the function $f_{q}(x):=\left(x^{q}-x\right) /(1-q)$ is concave. Since $\sum_{c} p(c \mid b)=1$, we have $p(c \mid b)^{q} \leq p(c \mid b)$ for $q \geq 1$. So, the sum with respect to $c$ obeys

$$
\sum_{c} p(c \mid b)^{q} f_{q}(p(a \mid b, c)) \leq \sum_{c} p(c \mid b) f_{q}(p(a \mid b, c)) \leq f_{q}\left(\sum_{c} p(c \mid b) p(a \mid b, c)\right)
$$

due to Jensen's inequality. As the numbers $p(c \mid b) p(a \mid b, c)=p(b, c) p(b)^{-1} p(a, b, c) p(b, c)^{-1}=p(a, b, c) p(b)^{-1}$ are summarized to $p(a, b) p(b)^{-1}=p(a \mid b)$, the right-hand side of Eq. (22) reads $f_{q}(p(a \mid b))$. Combining this with Eq. (21) then gives

$$
H_{q}(A \mid B, C) \leq \sum_{a b} p(b)^{q} f_{q}(p(a \mid b))=H_{q}(A \mid B)
$$

By a parallel argument, we easily have the case $n=1$, namely

$$
H_{q}(A \mid B) \leq H_{q}(A)
$$

The proof of Eq. (20) is completed by an extension with respect to $n$. The case $q=1$ can be recovered by repeating the above reasons with the concave function $f_{1}(x)=-x \ln x$.

Note that the formula (24) implies positivity of the mutual $q$-information (16) for all $q \geq 1$. There exists another form of the conditional $q$-entropy [41]. However, this form does not succeed some useful relations including the chain rule. Properties of both forms of the conditional $q$-entropy are discussed in the papers [41, 42]. The Fano inequality in terms of $q$-entropies and some of its applications are considered in Refs. [41, 43]. We will use the conditional $q$-entropy of order $q \geq 1$ for expressing inequalities of Bell's type. 


\section{MARGINAL SCENARIOS AND BELL INEQUALITIES}

The notion of marginal scenarios provides a general way to treat the noncontextuality of probability distributions 29, 34]. In a marginal problem, we ask whether a given family of marginal distributions for some set of random variables arises from some joint distribution of these variables 34]. Both Bell scenarios and contextuality scenarios can be unified in the following way [29]. Let $\left\{X_{1}, \ldots, X_{n}\right\}$ be a finite set of observables, and let $\mathcal{M}=\left\{S_{1}, \ldots, S_{|\mathcal{M}|}\right\}$ be a family of subsets $S_{i} \subseteq\left\{X_{1}, \ldots, X_{n}\right\}$. Such subsets are assumed to be comprised from commuting observables. In other words, each subset contains jointly measurable quantities. Hence, the two conditions $S \in \mathcal{M}$ and $S^{\prime} \subseteq S$ must imply $S^{\prime} \in \mathcal{M}$. When the family $\mathcal{M}$ obeys this implication, we call it "marginal scenario". For a formal consistency, the empty set $\emptyset$ is assumed to be included into $\mathcal{M}$.

From the physical viewpoint, one obtains some joint measurement statistics for each $S \in \mathcal{M}$. In real experiments, physicists usually deal with a collection of pairs of compatible observables. Suppose that $\{X, Y\} \in \mathcal{M}$. By $\operatorname{Pr}(x, y \mid X, Y)$, we denote the probability of obtaining the outcomes $x$ for $X$ and $y$ for $Y$ in their joint measurement. A similar notation will be used for more than two compatible observables. Note that the notation $\operatorname{Pr}(x, y \mid X, Y)$ assumes the specific physical context. In this sense, such probabilities should be distinguished from usual conditional probabilities. The introduced probabilities are used to pose formally criteria that given probabilistic model is not contextual [34]. An approach based on the algebraic language has been developed by Abramsky and Brandenburger [48].

Within an intuitive approach, we assign some hidden variable $\lambda$ to any physical model. It is assumed that this variable completely predetermines the future behavior. If the actual value of $\lambda$ was known, the probabilities $p_{X}(x \mid \lambda)$ of each observable $X$ are assumed to be independent of measurement statistics of all other observables [29]. Hence, for mutually compatible $X$ and $Y$ we can write

$$
\operatorname{Pr}(x, y \mid X, Y)=\sum_{\lambda} \varrho(\lambda) p_{X}(x \mid \lambda) p_{Y}(y \mid \lambda) .
$$

Here, unknown quantities $\varrho(\lambda)$ must obey $\varrho(\lambda) \geq 0$ and $\sum_{\lambda} \varrho(\lambda)=1$. Similarly to Eq. (25), we can deal with more than two compatible observables. The noncontextuality of a given model in marginal scenario $\mathcal{M}$ implies the existence of a joint probability distribution

$$
\operatorname{Pr}\left(x_{1}, \ldots, x_{n} \mid X_{1}, \ldots, X_{n}\right)=p\left(x_{1}, \ldots, x_{n}\right),
$$

which marginalizes to the model distributions for all $S \in \mathcal{M}$ [34, 48]. We then aim to decide, whether the considered probabilistic model obeys this criterion. It can be rewritten in terms of mean values or entropic functions.

Original Bell inequalities [7] were written in terms of mean values. Results of such a kind usually pertain to experiments, which probe entanglement between spacelike separated subsystems. The CHSH scenario is probably the most known setup. Let observables $A$ and $A^{\prime}$ be used for one subsystem, and let observables $B$ and $B^{\prime}$ be used for other. Both the pairs $\left\{A, A^{\prime}\right\}$ and $\left\{B, B^{\prime}\right\}$ are not jointly measurable. On the other hand, each element of $\left\{A, A^{\prime}\right\}$ is compatible with each element of $\left\{B, B^{\prime}\right\}$, since they are related to different subsystems. So, the marginal scenario includes the four singletons $\{A\},\left\{A^{\prime}\right\},\{B\},\left\{B^{\prime}\right\}$, and the four pairs $\{A, B\},\left\{A, B^{\prime}\right\},\left\{A^{\prime}, B\right\},\left\{A^{\prime}, B^{\prime}\right\}$. In the usual CHSH scenario, each of the observables has two possible outcomes. Let outcomes be rescaled to \pm 1 . The existence of a joint probability distribution for this scenario then leads to the CHSH inequality [25]

$$
\left\langle A B^{\prime}\right\rangle+\left\langle A^{\prime} B^{\prime}\right\rangle+\left\langle A^{\prime} B\right\rangle-\langle A B\rangle \leq 2 .
$$

Quantum mechanics predicts that the left-hand side of Eq. (27) can increase up to $2 \sqrt{2}$ [46]. Violations of Eq. (27) have been tested in experiments 26, 27]. Similarly, we formulate the scenario with arbitrary number of outcomes for observables. Assuming the existence of a joint probability distribution, Braunstein and Caves derived entropic inequality [22]

$$
H_{1}(A \mid B) \leq H_{1}\left(A \mid B^{\prime}\right)+H_{1}\left(B^{\prime} \mid A^{\prime}\right)+H_{1}\left(A^{\prime} \mid B\right) .
$$

The conditional entropy is asymmetric in its entries. The authors of Ref. [29] rewrite Eq. (28) in terms of the symmetrical mutual information, namely

$$
I_{1}\left(A: B^{\prime}\right)+I_{1}\left(A^{\prime}: B^{\prime}\right)+I_{1}\left(A^{\prime}: B\right)-I_{1}(A: B) \leq H_{1}\left(A^{\prime}\right)+H_{1}\left(B^{\prime}\right) .
$$

In a structure, the information-theoretic inequality (29) is similar to the usual CHSH inequality (27). When we apply Eq. (29) to test the nonlocality of a probability distribution, the following symmetries should be taken into account. By a permutation, the right-hand side of this inequality can be rewritten with every pair of compatible observables. 
Unlike the CHSH scenario, the KCBS scenario [28] is not associated with correlations between the measurements on different subsystems. The latter pertain to the measurements statistics for a single system. Here, we deal with five quantities $X_{1}, X_{2}, X_{3}, X_{4}, X_{5}$, such that each pair $\left\{X_{j}, X_{j+1}\right\}$ is jointly measurable. If quantities take values \pm 1 , then the existence of a joint probability distribution leads to the pentagram inequality [28]

$$
\sum_{j=1}^{5}\left\langle X_{j} X_{j+1}\right\rangle \geq-3
$$

The corresponding entropic formulation is expressed as 30$]$

$$
H_{1}\left(X_{1} \mid X_{5}\right) \leq H_{1}\left(X_{1} \mid X_{2}\right)+H_{1}\left(X_{2} \mid X_{3}\right)+H_{1}\left(X_{3} \mid X_{4}\right)+H_{1}\left(X_{4} \mid X_{5}\right) .
$$

The writers of Ref. 29] gave this inequality in other form known as the entropic Klyachko inequality. Advantages of entropic formulations are the following. First, they can handle any finite number of outcomes. Second, the entropic approach allows to study more realistic cases with detection inefficiencies [29]. Further, we will consider the following two models.

In the first model, two compatible observables are measured jointly by a single detector. By $\eta \in[0 ; 1]$, we quantify a detection efficiency. The no-click event is represented by additional outcome $(\varnothing, \varnothing)$. The new probability distribution includes the probabilities [29]

$$
\begin{aligned}
\operatorname{Pr}^{(\eta)}\left(x_{j}, x_{j+1} \mid X_{j}, X_{j+1}\right) & =\eta \operatorname{Pr}\left(x_{j}, x_{j+1} \mid X_{j}, X_{j+1}\right), \\
\operatorname{Pr}^{(\eta)}\left(\varnothing, \varnothing \mid X_{j}, X_{j+1}\right) & =1-\eta,
\end{aligned}
$$

where $x_{j}, x_{j+1} \in\{-1,+1\}$. This probability distribution marginalizes to the single-observable distribution

$$
\operatorname{Pr}^{(\eta)}\left(x_{j} \mid X_{j}\right)=\eta \operatorname{Pr}\left(x_{j} \mid X_{j}\right), \quad \operatorname{Pr}^{(\eta)}\left(\varnothing \mid X_{j}\right)=1-\eta .
$$

In this model, the no-click event occurs for both observables simultaneously with the probability (33). As shown in Ref. [29], the entropic Klyachko inequality merely scales by $\eta$. Thus, the inequality has a violation for all $\eta>0$. Violations take place in the same cases, for which the inequality with $\eta=1$ is violated. We will further show that these properties remain valid for the corresponding $q$-entropic inequalities.

In the second model, the joint measurement of $X_{j}$ and $X_{j+1}$ is performed by two detectors. We assume that each of detectors has an efficiency of $\eta \in[0 ; 1]$. It can be realized within some sequential scheme with a nondemolition measurement in the first detector [29]. For any jointly measurable pair, one writes the probabilities

$$
\begin{aligned}
\operatorname{Pr}^{(\eta \eta)}\left(x_{j}, x_{j+1} \mid X_{j}, X_{j+1}\right) & =\eta^{2} \operatorname{Pr}\left(x_{j}, x_{j+1} \mid X_{j}, X_{j+1}\right) \\
\operatorname{Pr}^{(\eta \eta)}\left(x_{j}, \varnothing \mid X_{j}, X_{j+1}\right) & =\eta(1-\eta) \operatorname{Pr}\left(x_{j} \mid X_{j}\right) \\
\operatorname{Pr}^{(\eta \eta)}\left(\varnothing, \varnothing \mid X_{j}, X_{j+1}\right) & =(1-\eta)^{2}
\end{aligned}
$$

where $\operatorname{Pr}^{(\eta \eta)}\left(\varnothing, x_{j+1} \mid X_{j}, X_{j+1}\right)$ is expressed similarly to Eq. (36). This probability distribution also marginalizes to the single-observable distribution (34). In this model, the required detection efficiency for witnessing quantum violations turned out be very high, $\eta \approx 0.995[29]$. In the following, we will consider this issue for $q$-entropic inequalities of the Bell type.

The CHSH and KCBS scenarios are both particular cases of the $n$-cycle [31, 32]. This notion is defined for any number $n \geq 3$ of observables $X_{1}, \ldots, X_{n}$ in a cyclic configuration. We demand that two observables $X_{j}$ and $X_{j+1}$ be jointly measurable for all $j=1, \ldots, n$. The complete characterization of the $n$-cycle scenario has been given for dichotomic observables, when possible outcomes are \pm 1 . Let each of $n$ factors $\gamma_{j}$ be either -1 or +1 , and let the total number of $\gamma_{j}=-1$ be odd. Then the noncontextuality implies [32]

$$
\sum_{j=1}^{n} \gamma_{j}\left\langle X_{j} X_{j+1}\right\rangle \leq n-2
$$

All $2^{n-1}$ inequalities of the form (38) characterize the $n$-cycle noncontextual polytope [32]. The CHSH inequality (27) is an example of Eq. (38) for $n=4$. Entropic formulations for the $n$-cycle scenario are examined in Refs. [29, 34].

\section{ENTROPIC INEQUALITIES FOR THE CHSH SCENARIO}

In this section, we formulate Bell's theorem in terms of the conditional $q$-entropies for the CHSH scenario. The $q$-entropic inequalities will be derived from the existence of joint probability distribution $p\left(a, b^{\prime}, a^{\prime}, b\right)$. This joint 
distribution should marginalize to the model distributions for all jointly measurable pairs. For instance, for the pair $\{A, B\}$ we have

$$
p(a, b)=\sum_{b^{\prime} a^{\prime}} p\left(a, b^{\prime}, a^{\prime}, b\right)
$$

and similarly for other jointly measurable subsets. Due to relations of the form (19), we write

$$
H_{q}(A, B) \leq H_{q}\left(A, B^{\prime}, A^{\prime}, B\right)=H_{q}\left(A \mid B^{\prime}, A^{\prime}, B\right)+H_{q}\left(B^{\prime} \mid A^{\prime}, B\right)+H_{q}\left(A^{\prime} \mid B\right)+H_{q}(B) .
$$

Here, the entropy $H_{q}\left(A, B^{\prime}, A^{\prime}, B\right)$ was expressed with respect to the chain rule (18). Subtracting $H_{q}(B)$ and using Eq. (14), one further obtains

$$
H_{q}(A \mid B) \leq H_{q}\left(A \mid B^{\prime}, A^{\prime}, B\right)+H_{q}\left(B^{\prime} \mid A^{\prime}, B\right)+H_{q}\left(A^{\prime} \mid B\right) .
$$

According to Lemma 2, for $q \geq 1$ we write

$$
H_{q}\left(A \mid B^{\prime}, A^{\prime}, B\right) \leq H_{q}\left(A \mid B^{\prime}\right), \quad H_{q}\left(B^{\prime} \mid A^{\prime}, B\right) \leq H_{q}\left(B^{\prime} \mid A^{\prime}\right) .
$$

Combining these relations with Eq. (41), we have arrived at the entropic inequality

$$
H_{q}(A \mid B) \leq H_{q}\left(A \mid B^{\prime}\right)+H_{q}\left(B^{\prime} \mid A^{\prime}\right)+H_{q}\left(A^{\prime} \mid B\right)
$$

which holds for $q \geq 1$. Predictions of quantum mechanics sometimes lead to a violation of Eq. (43). For $q=1$, this formula is reduced to the Braunstein-Caves inequality (28). Using the conditional $q$-entropies, we herewith obtained a one-parametric extension of the main result of Ref. [22]. In terms of the mutual $q$-information, for $q \geq 1$ we also have

$$
I_{q}\left(A: B^{\prime}\right)+I_{q}\left(A^{\prime}: B^{\prime}\right)+I_{q}\left(A^{\prime}: B\right)-I_{q}(A: B) \leq H_{q}\left(A^{\prime}\right)+H_{q}\left(B^{\prime}\right)
$$

It follows from Eq. (43) by immediate use of the definition (16). Similarly to Eq. (29), we should keep in mind possible permutations of the jointly measurable pairs in Eq. (44).

To observe violations of Eq. (43), we will deal with the four observables

$$
\begin{array}{ll}
\mathrm{A}=\vec{a} \cdot \overrightarrow{\boldsymbol{\sigma}} \otimes \mathbb{1}, & \mathrm{A}^{\prime}=\vec{a}^{\prime} \cdot \overrightarrow{\boldsymbol{\sigma}} \otimes \mathbb{1}, \\
\mathrm{B}=\mathbb{1} \otimes \vec{b} \cdot \overrightarrow{\boldsymbol{\sigma}}, & \mathrm{B}^{\prime}=\mathbb{1} \otimes \vec{b}^{\prime} \cdot \overrightarrow{\boldsymbol{\sigma}} .
\end{array}
$$

Here, the three-dimensional vectors $\vec{a}, \vec{a}^{\prime}, \vec{b}$, and $\vec{b}^{\prime}$ are unit; the $\overrightarrow{\boldsymbol{\sigma}}$ is the vector of Pauli matrices. Violations of Eq. (43) can be characterized by the quantity

$$
\mathcal{C}_{q}=H_{q}(A \mid B)-H_{q}\left(A \mid B^{\prime}\right)-H_{q}\left(B^{\prime} \mid A^{\prime}\right)-H_{q}\left(A^{\prime} \mid B\right)
$$

Following Ref. [22], we consider coplanar three-dimensional vectors $\vec{a}, \vec{b}^{\prime}$, $\vec{a}^{\prime}$, and $\vec{b}$, with the angles $\measuredangle\left(\vec{a}, \vec{b}^{\prime}\right)=$ $\measuredangle\left(\vec{b}^{\prime}, \vec{a}^{\prime}\right)=\measuredangle\left(\vec{a}^{\prime}, \vec{b}\right)=\gamma / 3$ and $\measuredangle(\vec{a}, \vec{b})=\gamma$. The initial state of two spin- $1 / 2$ systems is the state of zero total spin, namely

$$
|\Phi\rangle=\frac{1}{\sqrt{2}}(|0\rangle \otimes|1\rangle-|1\rangle \otimes|0\rangle) .
$$

In Eq. (48), the quantization axis is completely arbitrary. With such a choice, the characteristic quantity (47) can be rewritten as

$$
\mathcal{C}_{q}=H_{q}(A \mid B)-3 H_{q}\left(B^{\prime} \mid A^{\prime}\right)
$$

Here, the first term corresponds to the angle $\gamma$ between two unit vectors, and the second one corresponds to the angle $\gamma / 3$ between two unit vectors. Positive values of $\mathcal{C}_{q}$ imply violations of the locality hypothesis. It is useful to measure these positive values with a natural scale of entropic values. So, we will relate $\mathcal{C}_{q}$ with the number $\ln _{q} 2$, which represents the maximal binary $q$-entropy. That is, the results are reported in terms of the relative quantity

$$
\mathcal{R}_{q}:=\left(\ln _{q} 2\right)^{-1} \mathcal{C}_{q}
$$

Figure 1 presents violations of Eq. (43) for various $q \geq 1$. For comparison, we include the standard case $q=1$, when the maximum is equal to 0.2369 [22] and reached for $\gamma=0.9141$. With increase of $q$, the curve maximum goes 


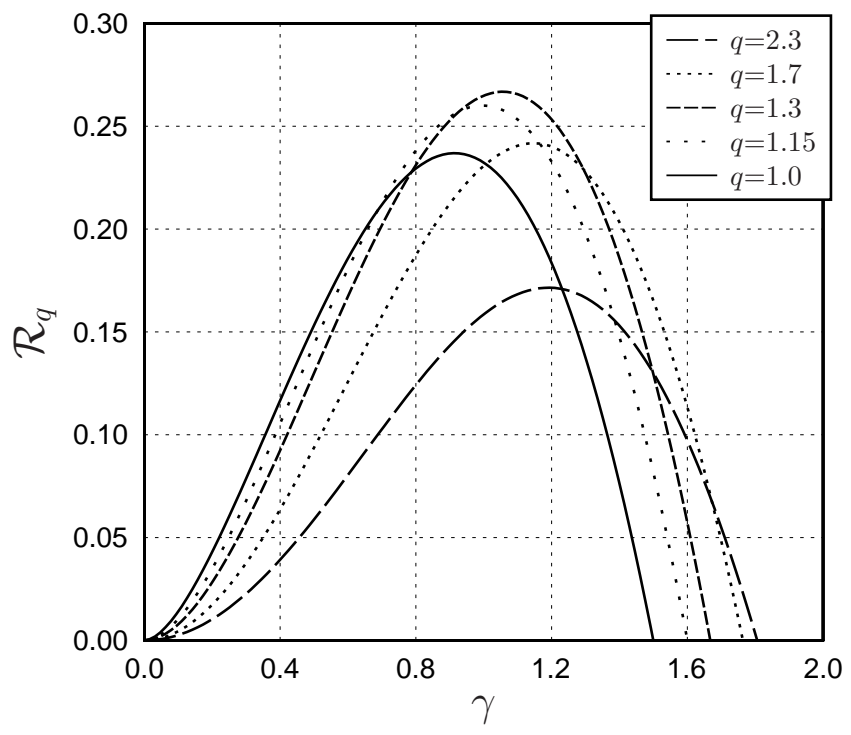

FIG. 1: The relative quantity $\mathcal{R}_{q}$ versus $\gamma$ in the case of Eq. (49) for $q=1.0 ; 1.15 ; 1.3 ; 1.7 ; 2.3$. For each $q$, only positive values of $\mathcal{R}_{q}$ are shown.

to larger values of $\gamma$. One shows some extension of the domain, for which $\mathcal{R}_{q}>0$. The inequality (43) is actually violated for one values of $q$ and is not violated for other, including $q=1$. Here, we can recall symmetries of Eqs. (29) and (44) with respect to permutations of the four measurable pairs. In the considered example, however, such permutations do not give new detectable cases for fixed $q$. Due to invariance of the state (48), we have relations of the form $H_{q}(A \mid B)=H_{q}(B \mid A)$, which depend only on the angle between two unit vectors. Thus, the $q$-entropic inequalities can detect the nonlocality of some probability distributions that cannot be detected by Eq. (28).

In general, entropic inequalities give only necessary criteria for the locality or noncontextualuity. In this sense, the $q$-entropic inequalities provide more powerful criteria. In the dichotomic CHSH scenario, the author of Ref. [49] has recently shown the following. Adding a shared randomness in the experimental setup, the Braunstein-Caves inequalities turn to be sufficient. Extending the depolarization protocol of Ref. [50], the sufficiency can be stated for any $n$-cycle with dichotomic outcomes. There exists also an argument without the depolarization procedure [49]. Using the entire family of $q$-entropic inequalities provides a complementary way, which can be essential with more than two outcomes.

It is easy to write the $q$-entropic inequalities for the $n$-cycle scenario. Here, each pair $\left\{X_{j}, X_{j+1}\right\}$ is jointly measurable. We suppose that there exist a joint probability distribution $p\left(x_{1}, x_{2}, \ldots, x_{n}\right)$, which marginalizes to twoobservable distributions of the form

$$
p\left(x_{1}, x_{2}\right)=\sum_{x_{j}: j \neq 1,2} p\left(x_{1}, x_{2}, \ldots, x_{n}\right)
$$

Assuming this, we extend Eq. (43) in the following way. For $q \geq 1$, one has

$$
H_{q}\left(X_{1} \mid X_{n}\right) \leq \sum_{j=1}^{n-1} H_{q}\left(X_{j} \mid X_{j+1}\right)
$$

This formula can be derived by means of obvious extension of the reasons from Eqs. (40)-(43). We refrain from presenting the details here. Using Eqs. (14) and (16), we could rewrite the inequality (52) with use of the joint $q$-entropies or the mutual $q$-informations. The former is essential in studying models of detection inefficiencies. We consider this issue in the next section. To compare Eq. (52) with predictions of quantum theory, we will use an immediate extension of Eq. (47). For $q=1$, such a quantity for the KCBS scenario was considered in Ref. [30]. The inequality (52) is then rewritten as $\mathcal{C}_{q} \leq 0$. If predictions of quantum mechanics do sometimes lead to strictly positive $\mathcal{C}_{q}$, then the noncontextuality hypothesis fails. In such a case, the quantity $\mathcal{C}_{q}$ characterizes an amount of violation of the inequality (52). As was argued in Ref. [30], violation of the inequality (52) implies violation of the corresponding pentagram inequality of Ref. [28], but the converse is not true. Such findings could be verified in appropriate experiments. 
TABLE I: The maximal values of $\mathcal{C}_{q}$ and $\mathcal{R}_{q}$ for several $q$.

\begin{tabular}{|c|c|c|c|c|c|c|c|c|c|c|c|c|}
\hline$q$ & 1.0 & 1.1 & 1.2 & 1.4 & 1.6 & 1.8 & 2.0 & 2.5 & 3.0 & 5.0 & 8.0 & 11.0 \\
\hline $\max \mathcal{C}_{q}$ & 0.0631 & 0.0779 & 0.0898 & 0.1049 & 0.1111 & 0.1113 & 0.1079 & 0.0924 & 0.0759 & 0.0383 & 0.0212 & 0.0146 \\
$\max \mathcal{R}_{q}$ & 0.0911 & 0.1164 & 0.1387 & 0.1733 & 0.1960 & 0.2093 & 0.2157 & 0.2143 & 0.2024 & 0.1632 & 0.1494 & 0.1462 \\
$\alpha_{\max }$ & 0.1698 & 0.1802 & 0.1880 & 0.1987 & 0.2051 & 0.2085 & 0.2099 & 0.2067 & 0.1982 & 0.1557 & 0.1205 & 0.1017 \\
$\theta_{\max }$ & 0.2366 & 0.2684 & 0.2943 & 0.3327 & 0.3585 & 0.3761 & 0.3880 & 0.4014 & 0.3996 & 0.3345 & 0.2639 & 0.2247 \\
\hline
\end{tabular}

\section{ENTROPIC INEQUALITIES FOR THE KCBS SCENARIO}

In this section, we examine $q$-entropic inequalities of the Bell type for the KCBS scenario. In the case $n=5$ and $q \geq 1$, the entropic inequality (52) reads

$$
H_{q}\left(X_{1} \mid X_{5}\right) \leq H_{q}\left(X_{1} \mid X_{2}\right)+H_{q}\left(X_{2} \mid X_{3}\right)+H_{q}\left(X_{3} \mid X_{4}\right)+H_{q}\left(X_{4} \mid X_{5}\right) .
$$

We also recall symmetries of such inequalities with respect to acceptable permutations. Following Refs. 229, 30], we consider projectors of the form $\left|X_{k}\right\rangle\left\langle X_{k}\right|$ with the eigenvectors

$$
\begin{aligned}
& \left|X_{1}\right\rangle=\frac{1}{\sqrt{2} \cos \alpha}\left(\begin{array}{c}
\sqrt{\cos 2 \alpha} \\
\sin \alpha \\
\cos \alpha
\end{array}\right), \quad\left|X_{2}\right\rangle=\left(\begin{array}{c}
0 \\
\cos \alpha \\
-\sin \alpha
\end{array}\right), \quad\left|X_{3}\right\rangle=\left(\begin{array}{l}
1 \\
0 \\
0
\end{array}\right), \\
& \left|X_{4}\right\rangle=\left(\begin{array}{c}
0 \\
\cos \alpha \\
\sin \alpha
\end{array}\right), \quad\left|X_{5}\right\rangle=\frac{1}{\sqrt{2} \cos \alpha}\left(\begin{array}{c}
\sqrt{\cos 2 \alpha} \\
\sin \alpha \\
-\cos \alpha
\end{array}\right),
\end{aligned}
$$

where $\alpha \in(0 ; \pi / 4)$. The five vectors satisfy orthogonality conditions

$$
\left\langle X_{1} \mid X_{2}\right\rangle=\left\langle X_{2} \mid X_{3}\right\rangle=\left\langle X_{3} \mid X_{4}\right\rangle=\left\langle X_{4} \mid X_{5}\right\rangle=\left\langle X_{5} \mid X_{1}\right\rangle=0 .
$$

The two projectors $\left|X_{k}\right\rangle\left\langle X_{k}\right|$ and $\left|X_{k+1}\right\rangle\left\langle X_{k+1}\right|$ are jointly measurable for all $k=1,2,3,4,5$. Eigenvalues 1 and 0 of the projector $\left|X_{k}\right\rangle\left\langle X_{k}\right|$ respectively correspond to outcomes "yes" and "no", when measured quantum state passes the test of being the state $\left|X_{k}\right\rangle$. The vectors (54)-(55) also obey $\left\langle X_{1} \mid X_{4}\right\rangle=\left\langle X_{5} \mid X_{2}\right\rangle$ and $\left\langle X_{1} \mid X_{3}\right\rangle=\left\langle X_{5} \mid X_{3}\right\rangle$. Further, we write the pre-measurement state as

$$
|\psi\rangle=\left(\begin{array}{c}
\sin \theta \\
\cos \theta \\
0
\end{array}\right),
$$

for which $\left\langle X_{1} \mid \psi\right\rangle=\left\langle X_{5} \mid \psi\right\rangle$ and $\left\langle X_{2} \mid \psi\right\rangle=\left\langle X_{4} \mid \psi\right\rangle$. Some intuitive reasons for such a configuration are briefly discussed in Ref. [30].

With the pre-measurement state $|\psi\rangle$, the observation of $X_{k}$ leads to the outcomes $x_{k}=1$ and $x_{k}=0$ with probabilities $\left|\left\langle X_{k} \mid \psi\right\rangle\right|^{2}$ and $1-\left|\left\langle X_{k} \mid \psi\right\rangle\right|^{2}$, respectively. According to the projection postulate, the normalized postmeasurement state is $\left|X_{k}\right\rangle$ for $x_{k}=1$ and

$$
\left(1-\left|\left\langle X_{k} \mid \psi\right\rangle\right|^{2}\right)^{-1 / 2}\left\{|\psi\rangle-\left|X_{k}\right\rangle\left\langle X_{k} \mid \psi\right\rangle\right\}
$$

for $x_{k}=0$. Hence, the context for next observations is determined. If the next observation is $X_{j}$, we calculate the conditional probabilities and, further, the corresponding entropy $H_{q}\left(X_{j} \mid X_{k}\right)$. In this quantum-mechanical way, one evaluates the characteristic quantity

$$
\mathcal{C}_{q}=H_{q}\left(X_{1} \mid X_{5}\right)-H_{q}\left(X_{1} \mid X_{2}\right)-H_{q}\left(X_{2} \mid X_{3}\right)-H_{q}\left(X_{3} \mid X_{4}\right)-H_{q}\left(X_{4} \mid X_{5}\right) .
$$

The inequality (53) implies $\mathcal{C}_{q} \leq 0$. The main result is its violations for certain values of the parameters $\alpha$ and $\theta$. We do not solve analytically the problem of finding a joint parametric domain, in which $\mathcal{C}_{q}>0$. For given parameters, however, the quantity $\mathcal{C}_{q}$ is easy to numerical estimation. Some numerical results are summarized below. Here, we will again use the quantity rescaled according to Eq. (50). In Table $\llbracket$, the maximal values of $\mathcal{C}_{q}$ and $\mathcal{R}_{q}$ are shown for several values of the parameter $q$. The values $\alpha_{\max }$ and $\theta_{\max }$, which correspond to the maximal violation, are given 


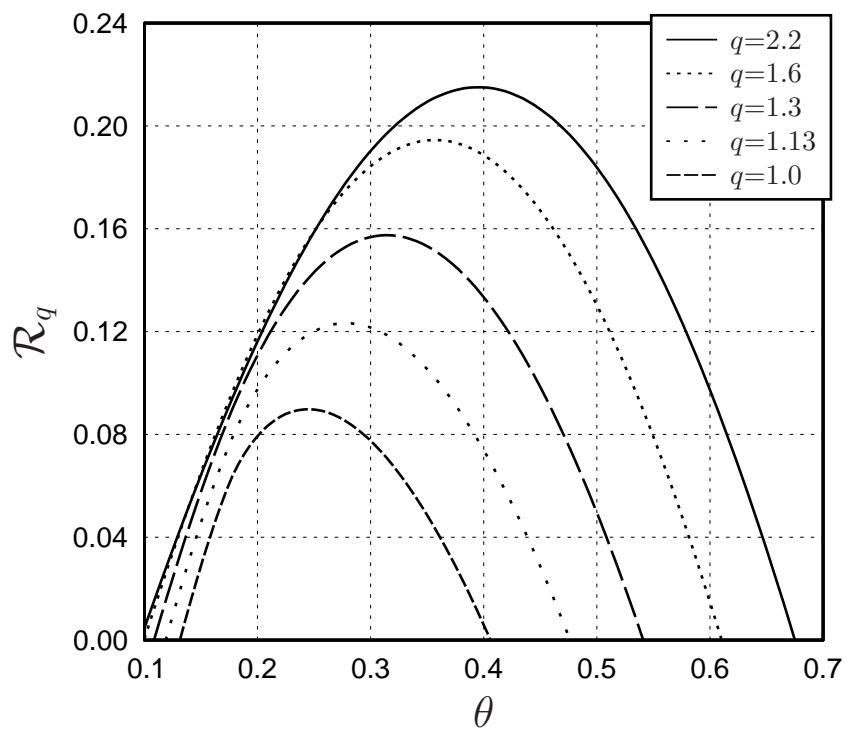

FIG. 2: The relative quantity $\mathcal{R}_{q}$ versus $\theta$ in the case of Eq. (59) for $\alpha=0.1885$ and $q=1.0 ; 1.13 ; 1.3 ; 1.6 ; 2.2$. For each $q$, only positive values of $\mathcal{R}_{q}$ are shown.

as well. In relative entropic size, the maximal violation of Eq. (553) is sufficiently large for all the presented values of $q$. The standard case $q=1$ was previously reported in Ref. [30]. For convenience of comparing with values $q>1$, we insert this case in the table. As we see in Table I the values $\alpha_{\max }$ and $\theta_{\max }$ depend on $q$. In given experimental setting, some fixed value of $\alpha$ and few values of $\theta$ would be rather available. On Fig. 2, a dependence of $\mathcal{R}_{q}$ on $\theta$ is given for $\alpha=0.1885$ and five values of the parameter $q$. We see that violation of Eq. (53) is significant for many values $q \geq 1$. Curves of Fig. 2 show the following important facts. First, the domain of $\theta$, in which $\mathcal{R}_{q}>0$, essentially increases with $q>1$. Hence, validity of Eq. (53) with some probabilistic model is not sufficient for its noncontextuality. Second, measurement statistics of the experiment with some fixed choice of $\theta$ does violate Eq. (53) for one values of $q$ and does not for other ones, including the standard case $q=1$. For instance, with $\theta=0.4765$ the inequality (53) is actually violated for $1.13<q$ and is not violated with $1 \leq q \leq 1.13$. In other words, the $q$-inequalities with properly chosen values of $q$ right detect the contextuality of some probability distributions that cannot be detected by Eq. (31) with the standard entropies. Thus, the family of $q$-entropic inequalities provides much more sensitive criteria for the contextuality. In the same experimental setup, therefore, we could test violation of the entire family of $q$-entropic inequalities of the Bell type. The obtained results can be regarded as an extension and development of theoretical findings of Refs. [29, 30].

We now consider $q$-entropic inequalities in the more realistic cases with detector inefficiencies. The writers of Ref. [29] considered these cases for inequalities with the Shannon entropies. It is convenient to rewrite Eq. (53) without conditional entropies. Using Eq. (14), we have $H_{q}\left(X_{j} \mid X_{j+1}\right)=H_{q}\left(X_{j}, X_{j+1}\right)-H_{q}\left(X_{j+1}\right)$. Then the formula (53) gives

$$
0 \leq \sum_{j=1}^{4} H_{q}\left(X_{j}, X_{j+1}\right)-H_{q}\left(X_{1}, X_{5}\right)-H_{q}\left(X_{2}\right)-H_{q}\left(X_{3}\right)-H_{q}\left(X_{4}\right)=-\mathcal{C}_{q}
$$

Due to detector inefficiencies, we obtain somewhat altered probability distributions. Hence, calculated entropies will somehow differ from the entropies involved in Eq. (60). The inequality (60) itself pertains to the inefficiency-free case, when $\eta=1$. In the single-detector model, probabilities are given by Eqs. (32) and (33) for the two-observable distribution and by Eq. (34) for the single-observable distribution. By $H_{q}^{(\eta)}\left(X_{j}, X_{j+1}\right)$ and $H_{q}^{(\eta)}\left(X_{j}\right)$, we denote the actual $q$-entropies calculated with such distributions. If the inequality (60) is valid, then the actual entropies satisfy the same formula, namely

$$
0 \leq \sum_{j=1}^{4} H_{q}^{(\eta)}\left(X_{j}, X_{j+1}\right)-H_{q}^{(\eta)}\left(X_{1}, X_{5}\right)-H_{q}^{(\eta)}\left(X_{2}\right)-H_{q}^{(\eta)}\left(X_{3}\right)-H_{q}^{(\eta)}\left(X_{4}\right) .
$$


Indeed, from Eq. (7) we immediately write

$$
\begin{aligned}
H_{q}^{(\eta)}\left(X_{j}, X_{j+1}\right) & =\eta^{q} H_{q}\left(X_{j}, X_{j+1}\right)+h_{q}(\eta), \\
H_{q}^{(\eta)}\left(X_{j}\right) & =\eta^{q} H_{q}\left(X_{j}\right)+h_{q}(\eta) .
\end{aligned}
$$

Substituting these expressions, the inequality (61) is recast as Eq. (60) multiplied by factor $\eta^{q}$. In the single-detector model, therefore, the noncontextuality hypothesis leads to the family of $q$-entropic inequalities of the form (61) with $q \geq 1$. The following points should be emphasized. First, in the considered model violations of Eq. (61) are irrelevant to the detection efficiency $\eta>0$. Second, for fixed $q$ the maximal violation takes place in the same cases, for which the inefficiency-free inequality is maximally violated. For the observables (54)-(55) and the state (57), some cases of the maximal violation were given above in Table \. In this regard, $q$-entropic inequalities of the Bell type succeed properties of more usual inequalities in terms of the Shannon entropies.

In the second model of detector inefficiencies, probabilities of the two-observable distribution are expressed by Eqs. (35), (36), and (37). These two-observable distributions also marginalize to the single-observable distributions of the form (34). By $H_{q}^{(\eta \eta)}\left(X_{j}, X_{j+1}\right)$ and $H_{q}^{(\eta \eta)}\left(X_{j}\right)$, we denote the actual $q$-entropies in the considered model of inefficiencies. Using Eq. (8), we obtain

$$
\begin{aligned}
H_{q}^{(\eta \eta)}\left(X_{j}, X_{j+1}\right) & =\eta^{2 q} H_{q}\left(X_{j}, X_{j+1}\right)+\eta^{q}(1-\eta)^{q}\left(H_{q}\left(X_{j}\right)+H_{q}\left(X_{j+1}\right)\right) \\
& +\left(\eta^{q}+(1-\eta)^{q}+1\right) h_{q}(\eta) .
\end{aligned}
$$

On the other hand, the entropy $H_{q}^{(\eta \eta)}\left(X_{j}\right)$ is equal to the right-hand side of Eq. (63). For brevity, we introduce the quantity

$$
\mathcal{C}_{q}^{(\eta \eta)}:=-\sum_{j=1}^{4} H_{q}^{(\eta \eta)}\left(X_{j}, X_{j+1}\right)+H_{q}^{(\eta \eta)}\left(X_{1}, X_{5}\right)+H_{q}^{(\eta \eta)}\left(X_{2}\right)+H_{q}^{(\eta \eta)}\left(X_{3}\right)+H_{q}^{(\eta \eta)}\left(X_{4}\right)
$$

In the inefficiency-free case, when $\eta=1$, this term coincides with the characteristic quantity (59). Using Eqs. (63) and (64), we represent the right-hand side of Eq. (65) as

$$
\begin{aligned}
\mathcal{C}_{q}^{(\eta \eta)} & =\eta^{2 q} \mathcal{C}_{q}-\Delta_{q}(\eta) \\
\Delta_{q}(\eta) & =\eta^{q}\left(\eta^{q}+2(1-\eta)^{q}-1\right)\left(H_{q}\left(X_{2}\right)+H_{q}\left(X_{3}\right)+H_{q}\left(X_{4}\right)\right) \\
& +3\left(\eta^{q}+(1-\eta)^{q}\right) h_{q}(\eta)
\end{aligned}
$$

The second summand in the right-hand side of Eq. (67) is positive. For $q>1$, the factor $\eta^{q}+2(1-\eta)^{q}-1$ is negative for some values of $\eta$ near 1 from below. So, the first summand in the right-hand side of Eq. (67) can take positive or negative values. The noncontextuality inequality (60) implies $\mathcal{C}_{q} \leq 0$. Using measurement statistics, however, we actually deal with the quantity (65). Suppose that measurement data have lead to the result $\mathcal{C}_{q}^{(\eta \eta)}>0$. Generally, one cannot conclude $\mathcal{C}_{q}>0$ without the following. We must confide that the violating term $\eta^{2 q} \mathcal{C}_{q}$ is sufficiently large in comparison with the additional term (67). To compare these terms, we introduce their ratio

$$
r_{q}(\eta):=\eta^{-2 q} \mathcal{C}_{q}^{-1}\left|\Delta_{q}(\eta)\right|
$$

which is related to the case $\mathcal{C}_{q}>0$. To obtain concrete estimates of $\eta$, we have found numerically the ratio (68) in the cases of maximal violation, which are shown in Table [1. In these cases, the additional term (67) turns to be nonnegative for all $\eta \in[0 ; 1]$. Then the experimental result $\mathcal{C}_{q}^{(\eta \eta)}>0$ would witness $\mathcal{C}_{q}>0$, i.e. quantum violations of the noncontextuality hypothesis. However, large values of $\Delta_{q}(\eta)$ can prevent this, even if the theoretical violation is maximal. Therefore, used detection schemes should provide the ratio (68) to be sufficiently small.

We have calculated $r_{q}(\eta)$ versus $\eta$ for all the cases listed in Table $\square$. With respect to $\eta$, we especially focus an attention on values, which are very close to 1 from below. As calculations show, for fixed $q$ the ratio $r_{q}(\eta)$ decreases with such $\eta$ almost linearly, up to the inefficiency-free value $r_{q}(1)=0$. Due to almost linear dependence, we can describe each case by the value of Eq. (68) for some suitably chosen $\eta$, say, for $\eta=0.99$. For estimation purposes, one then writes approximate formula

$$
r_{q}(\eta) \approx 10^{2} r_{q}(0.99)(1-\eta)
$$

which is appropriate within a range of linear behavior. In Table II the value $r_{q}(0.99)$ is presented for the cases of maximal violation, which are given above in Table I. Initially, this value significantly decreases with $q>1$. Further, it 
TABLE II: The values of the ratio (68) for $\eta=0.99$ and several $q$ in some cases of maximal violation.

\begin{tabular}{|c|c|c|c|c|c|c|c|c|c|c|c|c|}
\hline$q$ & 1.0 & 1.1 & 1.2 & 1.4 & 1.6 & 1.8 & 2.0 & 2.5 & 3.0 & 5.0 & 8.0 & 11.0 \\
\hline$r_{q}(0.99)$ & 2.0400 & 1.8593 & 1.3065 & 0.7764 & 0.5461 & 0.4294 & 0.3641 & 0.2944 & 0.2793 & 0.3845 & 0.6227 & 0.8656 \\
\hline
\end{tabular}

becomes increasing for sufficiently large $q$. In general, the required detection efficiency is very high. This conclusion concurs with the efficiency $\eta \approx 0.995$, which was claimed in Ref. [29] for relations with the Shannon entropies. A novel point is that, for given $\eta$, the ratio (68) essentially depends also on $q$. Among $q$-entropic inequalities for the KCBS scenario with observables (54)-(55), the choice $q=2$ can be recognized as very appropriate. First, the value of $\max \mathcal{C}_{q}$ for $q=2$ is almost maximal in comparison with other (see Table I). Second, the ratio (68) in the second model of detection inefficiencies is sufficiently small for $\eta>0.99$ (see Table II). Third, properties of the $q$-entropies are mathematically simpler just in the case $q=2$. Some of these properties were considered in Ref. [44]. With the family of $q$-entropic inequalities, therefore, we can obtain new possibilities for analyzing measurement data with detection insufficiencies.

\section{CONCLUSIONS}

In the paper, we have expressed Bell's theorem in terms of the conditional $q$-entropies of order $q \geq 1$. Formally, the presented inequalities are based on several useful properties of the conditional $q$-entropy. One of them is the well-known chain rule. Other required properties are proved as Lemmas 1 and 2. The latter result is combined with the chain rule in deriving new $q$-entropic inequalities of Bell's type. The statement of Lemma 1 is used to study the more realistic cases with detection inefficiencies. The result of Lemma 2 holds for $q \geq 1$ and generalizes analogous property of the standard conditional entropy. From the physical viewpoint, the noncontextuality hypothesis is a key ingredient of the derivation. Assuming the existence of a joint probability distribution for the outcomes of all observations, we have arrived at a principal conclusion. Namely, the corresponding conditional $q$-entropies of order $q \geq 1$ should satisfy inequalities of the form (52). This claim generalizes the previous entropic formulations of Bell's theorem. In particular, the inequality (43) is a $q$-parametric extension of the Braunstein-Caves inequality [22]. Thus, we have shown that the noncontextuality hypothesis leads to the entire family of $q$-entropic inequalities of Bell's type. It turns out that these inequalities are incompatible with the predictions of quantum mechanics for many values of the parameters.

With the standard conditional entropy, violations of entropic Bell inequalities were examined for the CHSH scenario in Refs. [22, 29] and for the KCBS scenario in Refs. [29, 30]. We have explicitly considered violations of the $q$-entropic inequalities in both the scenarios. The following principal conclusions can be made. First, the derived $q$-entropic inequalities allow to expand significantly a class of probability distributions, for which the nonlocality or contextuality are testable in this way. Using the $q$-entropic inequalities is an alternative to the approach with adding some shared randomness [49]. Second, the $q$-entropic inequalities are expedient in analyzing cases with detection inefficiencies. In the single-detector model, features of the $q$-entropic inequalities are quite similar to features of usual inequalities in terms of the Shannon entropies. In the two-detector model, the use of the $q$-entropic inequalities can allow to reduce an amount of required detection efficiency. The obtained conclusions for various values $q \geq 1$ could be tested in the experiment. For the conventional CHSH inequality in terms of average values, quantum violation is limited by the Tsirel'son bound. It would be interesting to obtain upper bounds on possible violations of $q$-entropic inequalities of Bell's type. Due to the role of entangled states in quantum information processing, theoretical results of such a kind may also have a practical significance.

\section{Acknowledgments}

I am grateful to Dr. Tobias Fritz and Dr. Rafael Chaves for useful correspondence and to anonymous referee for constructive criticism.

[1] M. A. Nielsen and I. L. Chuang (2000), Quantum Computation and Quantum Information, Cambridge University Press (Cambridge). 
[2] E. Schrödinger (1935), Die gegenwärtige situation in der quantenmechanik, Naturwissenschaften 23, 807-812, 823-828, 844-849; the translation is reprinted in J. A. Wheeler, W. H. Zurek, eds. (1983), Quantum Theory and Measurement, 152-167, Princeton University Press (Princeton).

[3] A. Einstein, B. Podolsky, and N. Rosen (1935), Can quantum-mechanical description of physical reality be considered complete? Phys. Rev. 47, 777-780; reprinted in J. A. Wheeler, W. H. Zurek, eds. (1983), Quantum Theory and Measurement, 138-141, Princeton University Press (Princeton).

[4] D. Bohm (1951), Quantum Theory, Prentice-Hall, Englewood Cliffs (New Jersey).

[5] M. J. W. Hall (2010), Local deterministic model of singlet state correlations based on relaxing measurement independence, Phys. Rev. Lett. 105, 250404.

[6] J. Barrett and N. Gisin (2011), How much measurement independence is needed to demonstrate nonlocality?, Phys. Rev. Lett. 105, 100406.

[7] J. S. Bell (1964), On the Einstein-Podolsky-Rosen paradox, Physics 1, 195-200.

[8] A. Zeilinger (1999), Experiment and the foundations of quantum physics, Rev. Mod. Phys. 71, S288-S297.

[9] A. Acín, N. Gisin, L. Masanes, and V. Scarani (2004), Bell's inequalities detect efficient entanglement, Int. J. Quant. Inf. 2, 23-32.

[10] A. Acín, N. Gisin, and V. Scarani (2003), Security bounds in quantum cryptography using d-level systems, Quantum Information \& Computation 3, 563-580.

[11] S. Pironio, A. Acín, S. Massar, A. Boyer de la Giroday, D. N. Matsukevich, P. Maunz, S. Olmschenk, D. Hayes, L. Luo, T. A. Manning, and C. Monroe (2010), Random numbers certified by Bell's theorem, Nature 464, 1021-1024.

[12] A. J. Leggett and A. Garg (1985), Quantum mechanics versus macroscopic realism: Is the flux there when nobody looks? Phys. Rev. Lett. 54, 857-860.

[13] C. Emary, N. Lambert, and F. Nori (2013), Leggett-Garg inequalities, arXiv:1304.5133 [quant-ph].

[14] A. R. Usha Devi, H. S. Karthik, Sudha, and A. K. Rajagopal (2013), Macrorealism from entropic Leggett-Garg inequalities, Phys. Rev. A 87, 052103.

[15] J. S. Bell (1966), On the problem of hidden variables in quantum mechanics, Rev. Mod. Phys. 38, $447-452$.

[16] S. Kochen and E. P. Specker (1967), The problem of hidden variables in quantum mechanics, J. Math. Mech. 17, 59-87.

[17] N. D. Mermin (1993), Hidden variables and the two theorems of John Bell, Rev. Mod. Phys. 65, $803-815$.

[18] O. Oreshkov, F. Costa, and Č. Brukner (2012), Quantum correlations with no causal order, Nature Communications 3, 1092. DOI: $10.1038 /$ ncomms2076

[19] D. M. Greenberger, M. A. Horne, A. Shimony, and A. Zeilinger (1990), Bell's theorem without inequalities, Am. J. Phys. 58, 1131-1143.

[20] A. Acín, G. Vidal, and J. I. Cirac (2003), On the structure of a reversible entanglement generating set for three-partite states, Quantum Information \& Computation 3, 55-63.

[21] A. Salles, D. Cavalcanti, A. Acín, D. Pérez-García, and M. M. Wolf (2010), Bell inequalities from multilinear contractions, Quantum Information \& Computation 10, 0703-0719.

[22] S. L. Braunstein and C. M. Caves (1988), Information-theoretic Bell inequalities, Phys. Rev. Lett. 61, $662-665$.

[23] N. J. Cerf and C. Adami (1997), Entropic Bell inequalities, Phys. Rev. A 55, 3371-3374.

[24] R. Renner (2005), Security of quantum key distribution. PhD thesis, arXiv:quant-ph/0512258

[25] J. F. Clauser, M. A. Horne, A. Shimony, and R. A. Holt (1969), Proposed experiment to test local hidden-variable theories, Phys. Rev. Lett. 23, 880-884.

[26] A. Aspect, P. Grangier, and G. Roger (1982), Experimental realization of Einstein-Podolsky-Rosen-Bohm gedankenexperiment, Phys. Rev. Lett. 49, 91-94.

[27] A. Aspect, J. Dalibard, and G. Roger (1982), Experimental tests of Bell's inequalities using time-varying analyzers, Phys. Rev. Lett. 49, 1804-1807.

[28] A. A. Klyachko, M. A. Can, S. Binicioğlu, and A. S. Shumovsky (2008), Simple test for hidden variables in spin-1 systems, Phys. Rev. Lett. 101, 020403.

[29] R. Chaves and T. Fritz (2012), Entropic approach to local realism and noncontextuality, Phys. Rev. A 85, 032113.

[30] P. Kurzyński, R. Ramanathan, and D. Kaszlikowski (2012), Entropic test of quantum contextuality, Phys. Rev. Lett. 109, 020404.

[31] Y.-C. Liang, R. W. Spekkens, and H. M. Wiseman (2011), Specker's parable of the over-protective seer: A road to contextuality, nonlocality and complementarity, Phys. Rep. 506, 1-39.

[32] M. Araújo, M. T. Quintino, C. Budroni, M. T. Cunha, and A. Cabello (2013), All noncontextuality inequalities for the n-cycle scenario, Phys. Rev. A 88, 022118

[33] G. Borges, M. Carvalho, P.-L. de Assis, J. Ferraz, M. Araújo, A. Cabello, M. T. Cunha, and S. Pádua (2013), Experimental test of the quantum violation of the noncontextuality inequalities for the n-cycle scenario, arXiv:1304.4512 [quant-ph].

[34] R. Chaves and T. Fritz (2013), Entropic inequalities and marginal problems, IEEE Trans. Inf. Theory 59, 803-817.

[35] A. Rényi (1961), On measures of entropy and information, Proc. 4th Berkeley Symposium on Mathematical Statistics and Probability, 547-561, University of California Press: Berkeley-Los Angeles.

[36] C. Tsallis (1988), Possible generalization of Boltzmann-Gibbs statistics, J. Stat. Phys. 52, 479-487.

[37] J. Havrda and F. Charvát (1967), Quantification methods of classification processes: concept of structural $\alpha$-entropy, Kybernetika 3, 30-35.

[38] G. A. Raggio (1995), Properties of q-entropies, J. Math. Phys. 36, 4785-4791.

[39] I. Bengtsson and K. Życzkowski (2006), Geometry of Quantum States: An Introduction to Quantum Entanglement, Cam- 
bridge University Press (Cambridge).

[40] T. M. Cover and J. A. Thomas (1991), Elements of Information Theory, John Wiley \& Sons (New York).

[41] S. Furuichi (2006), Information-theoretical properties of Tsallis entropies, J. Math. Phys. 47, 023302.

[42] A. E. Rastegin (2012), Convexity inequalities for estimating generalized conditional entropies from below, Kybernetika 48, 242-253.

[43] A. E. Rastegin (2013), Bounds of the Pinsker and Fannes types on the Tsallis relative entropy, Math. Phys. Anal. Geom. 16, 213-228.

[44] I. Vajda (1968), Bounds of the minimal error probability on checking a finite or countable number of hypotheses, Problemy Peredači Informacii 4, 9-19 (in Russian); translated as Problems of Information Transmission 4, 6-14.

[45] T. Yamano (2001), Information theory based on nonadditive information content, Phys. Rev. E $63,046105$.

[46] B. S. Tsirel'son (1980), Quantum generalizations of Bell's inequality, Lett. Math. Phys. 4, 93-100.

[47] E. Wakakuwa and M. Murao (2012), The chain rule implies Tsirelson's bound: an approach from generalized mutual information, New J. Phys. 14, 113037.

[48] S. Abramsky and A. Brandenburger (2011), The sheaf-theoretic structure of non-locality and contextuality, New J. Phys. 13, 113036.

[49] R. Chaves (2013), Entropic inequalities as a necessary and sufficient condition to noncontextuality and locality, Phys. Rev. A 87, 022102.

[50] L. Masanes, A. Acín, and N. Gisin (2006), General properties of nonsignaling theories, Phys. Rev. A 73, 012112. 